\title{
Antenatal physical activity counseling among healthcare providers
}

\author{
Jenn Leiferman', Margaret Gutilla ${ }^{2}$, James Paulson ${ }^{3}$, Jim Pivarnik ${ }^{4}$ \\ ${ }^{1}$ Department of Community and Behavioral Health, Colorado School of Public Health, Aurora, USA \\ ${ }^{2}$ Department of Epidemiology, Colorado School of Public Health, Aurora, USA \\ ${ }^{3}$ Old Dominion University, Norfolk, USA \\ ${ }^{4}$ Michigan State University, East Lansing, USA \\ Email: jenn.leiferman@ucdenver.edu
}

Received 10 August 2012; revised 9 September 2012; accepted 18 September 2012

\begin{abstract}
Objective: Pregnant women often report a lack of knowledge concerning the safety of exercising during pregnancy. Healthcare providers play an integral role in providing pregnant women with the necessary knowledge to promote antenatal physical activity. Thus, the objective of this study was to assess healthcare providers' beliefs, attitudes, knowledge and practices related to antenatal physical activity counseling. Study Design: 188 Providers (i.e. obstetricians, midwives, and family medicine physicians) completed a 39 closed-item survey. Characteristics among healthcare providers' physical activity counseling practices as well as belief, attitudes and knowledge were explored. Results: The majority of all providers agreed that physical activity during pregnancy will result in numerous improved health outcomes for mother and baby. Approximately half of the providers $(48 \%, n=$ 89) were not familiar with the current national guides recommending that women free of obstetric complications should engage in at least 150 minutes of exercise per week. Only $43 \%$ of providers believed their patients followed the advice they are given about physical activity. Over half of the providers reported that they provide in-office physical activity counseling, and FMs provide individualized counseling less often than OBs and CNMs (i.e. 33\%, 60\%, and 65\%, respectively; $p=0.0014)$. Importantly, $17 \%(n=31)$ of providers reported that they never received professional training in antenatal physical activity counseling and of those that did receive training, $69 \%(n=$ 107) claimed their training was "fair" or "poor". Conclusion: Findings from the pre- sent study demonstrate a need for further continuing education op-
\end{abstract}

"Disclosure: None of the authors have a conflict of interest.

This work is support by grant AHRQ \#1R03HS018595-01A1.

The present study examined healthcare providers' current attitudes, beliefs and practices related to antenatal physical activity counseling. portunities on the current national guidelines on antenatal physical activity.

Keywords: Exercise; Pregnancy; Physical Activity; Primary Care

\section{INTRODUCTION}

Despite the fact that the American Congress of Obstetricians and Gynecologists (ACOG) recommends pregnant women who are free of obstetrical complications engage in leisure-time physical activity (LTPA), women who are pregnant engage in less LTPA than their nonpregnant counterparts [1,2]. Specifically, Evenson et al. 2004 found that only $15.1 \%$ of pregnant women were engaging in the recommended amounts of LTPA versus $26.1 \%$ of nonpregnant women. Moreover, LTPA levels often decrease throughout gestation [3]. Given the lower rates of LTPA among pregnant women, coupled with the many maternal benefits associated with antenatal LTPA such as increased fitness [4], prevention of excess weight gain [5], low back pain [6], and lower anxiety and depressive symptomatology [7], there is a definite need to promote engagement in antenatal LTPA [3,8].

Pregnancy is an opportune time for women to modify their health behaviors. This time can be thought of as an "external motivator" to elicit behavior change in order to protect the health of the fetus as well as the mother's own health. Providing pregnant women with the necessary knowledge to promote engagement in physical activity can not only positively impact antenatal health outcomes (e.g. excessive antenatal weight gain, antenatal mood disorders, among others) but also those that occur in the postpartum period and beyond [9].

Engaging in regular physical activity decreases an individual's risk of overweight and associated chronic diseases such as, cardiovascular disease, type 2 diabetes mellitus, osteoporosis, obesity, and certain types of cancer 
(see review [10]). In particular, participation in walking ( 3 times a week for 45 min or 5 times a week for 30 min) is linked to reduced risk of coronary events in women [11]. Thus, promoting physical activity during pregnancy may not only reduce the risk of excessive antenatal weight gain, but also reduced risk of future overweight and chronic disease.

Given the demonstrated benefits of antenatal physical activity, why do many women fail to engage in LTPA? Pregnant women often report a lack of knowledge concerning the safety of exercising during pregnancy and believe that if they received information related to how to safely and effectively exercise during pregnancy it would facilitate their engagement in physical activity [12-14]. Incorrect information or complete absence of counseling from healthcare providers (HCPs) may compound this problem. This is unfortunate, given that brief physical activity counseling by HCPs increases the likelihood that patients will engage in physical activity $[15,16]$. Moreover, recent research suggests that the majority of women indicate that their HCPs have the most influence on their beliefs regarding physical activity [16]. Even with clear evidence delineating the benefits of antenatal physical activity counseling by HCPs on numerous maternal and child health outcomes, much of the healthcare system has not promoted effectively the concept that LTPA can be used to prevent and treat disease.

To date, there is a dearth of literature examining the extent to which HCPs provide brief counseling on antenatal physical activity to their pregnant patients. Much of the extant literature has been narrow in scope and limited to small, convenience samples [17-19] or examines primary care systems outside of the US, such as Australia [20]. For example, Entin and Munhall administered an 18-item survey to 83 obstetricians in private or small group practices in the US. Approximately half of the obstetricians surveyed reported that they do not routinely discuss exercise with their patients, and that most are hesitant to advise sedentary women to start exercise [17]. Bauer et al. (2004) surveyed 60 practicing physicians in Michigan and found that the majority believe exercise is beneficial and that they recommend exercise to their patients; however, it appears that not all are aware, or follow, current ACOG recommendations [18]. Recently Hughes et al. 2011 surveyed HCPs in general practice, community nursing and pharmacy to determine continuing education needs related to perinatal physical activity counseling. Results suggested that the majority of providers agree that physical activity guidance is important and recommend that future continuing education efforts be tailored according to type of primary care training [20]. Moreover, a large surveillance study which focused primarily on obesity prevention and weight gain counseling among primary care HCPs in the US provides some insight as to prevalence rates of antenatal physical activity counseling but fails to provide a comprehensive assessment of the potential predictors of counseling such as HCPs' attitudes, beliefs and perceived barriers [21]. A more comprehensive assessment of change agents (e.g., attitudes, beliefs, perceived barriers) would provide the critical information needed to inform future intervention efforts designed to promote antenatal physical activity counseling among HCPs. The present study addresses this gap in the literature by providing a more comprehensive assessment of potential factors (i.e. beliefs, attitudes, knowledge, self-efficacy, barriers) related to antenatal physical activity counseling among a diverse sample of HCPs who provide obstetric care. Data obtained from this study will help inform future interventions aimed at enhancing antenatal physical activity counseling in primary care settings.

\section{MATERIALS AND METHODS}

Prior to conducting this investigation, the study protocol was approved by the Colorado Multiple Institutional Review Board. A HCP was eligible to participate in the study if he/she was a physician (i.e., practicing in obstetrics or family medicine) or a certified nurse midwife (CNM) who was currently practicing in the DenverAurora Metropolitan Statistical Area (DAMSA). Eligible HCPs who met the study inclusion criteria were identified through local hospital and clinical directories and local chapters of the American Academy of Family Medicine, Certified Nurse Midwives, and the American College of Obstetricians and Gynecologists. Our initial search yielded 1210 names and included all MDs practicing obstetrics, all family doctors, and CNMs in the DAMSA. From this list, 59 obstetricians (OBs) and 7 CNMs were removed from the sample due to incorrect contact information. Additionally, 569 family medicine physicians (FMP) were eliminated due to either not providing obstetric care for their patients (54\%) or incorrect current contact information (19\%).

A total of 575 HCPs (i.e. 259 OBs; 104 CNMs, and 212 FMPs) were invited to participate in the study. A pre-notification of the study was sent via email or facsimile to all eligible HCPs to increase awareness of the upcoming invitation to participate in the survey. Within one week of the pre-notification, each eligible HCP was sent an email and/or facsimile that briefly described the survey and that contained a web link to the site where the survey would be hosted. In cases in which a HCP did not have access to email or fax, a paper invitation (containing the web address to the survey) was mailed to the individual via regular postal service. Additional followup attempts were made by email and postal mail notifications by the research team as well as by the HCPs respective organizations including the Colorado section of 
ACOG, Colorado section of the American College of Nurse Midwives, and the Colorado Academy of Family Physicians. Participants were given the opportunity to complete the survey by mail as well as by web. The majority of respondents (i.e. 55\%) responded by paper survey.

The final sample comprised 188 HCPs currently practicing in one of three specialties (i.e. family medicine, obstetrics, and midwifery) in the DAMSA. The sample included 91 obstetricians, 40 midwives, and 57 family medicine physicians, resulting in an overall response rate of $32.7 \%$. There were no significant differences between responders and nonresponders on age and county in which the HCP worked. However, there was a significant difference in response rate by gender among obstetricians only , with female obstetricians more likely than male obstetricians to respond $(\mathrm{p}=.014)$. Demographics for the full sample and by specialty are shown in Table 1.

Table 1. Sample description by specialty.

\begin{tabular}{|c|c|c|c|c|c|c|c|c|}
\hline \multirow{2}{*}{ Gender } & \multicolumn{2}{|c|}{$\begin{array}{c}\text { Full Sample } \\
\text { (N = 188) }\end{array}$} & \multicolumn{2}{|c|}{$\begin{array}{c}\text { OB } \\
(\mathrm{N}=91)\end{array}$} & \multicolumn{2}{|c|}{$\begin{array}{c}\text { FMP } \\
(\mathrm{N}=57)\end{array}$} & \multicolumn{2}{|c|}{$\begin{array}{c}\text { CNM } \\
(\mathrm{N}=40)\end{array}$} \\
\hline & $\mathbf{n}$ & (\%) & $\mathbf{n}$ & $(\%)$ & $\mathbf{n}$ & (\%) & $\mathbf{n}$ & $(\%)$ \\
\hline Male & 55 & (29.3\%) & 22 & (24.2\%) & 32 & (56.1\%) & 1 & $(2.5 \%)$ \\
\hline Female & 133 & (70.7\%) & 69 & (75.8\%) & 25 & (43.9\%) & 39 & (97.5\%) \\
\hline \multicolumn{9}{|l|}{ Race } \\
\hline White & 171 & (91.9\%) & 81 & (90.0\%) & 51 & (91.1\%) & 39 & (97.5\%) \\
\hline African American & 4 & $(2.2 \%)$ & 2 & $(2.2 \%)$ & 2 & (3.6\%) & 0 & $(0 \%)$ \\
\hline Asian & 8 & $(4.3 \%)$ & 5 & $(5.6 \%)$ & 2 & (3.6\%) & 1 & $(2.5 \%)$ \\
\hline American Indian & 1 & $(0.5 \%)$ & 1 & $(1.1 \%)$ & 0 & $(0 \%)$ & 0 & $(0 \%)$ \\
\hline Other & 2 & (1.1\%) & 1 & $(1.1 \%)$ & 1 & $(1.7 \%)$ & 0 & $(0 \%)$ \\
\hline \multicolumn{9}{|l|}{ Ethnicity } \\
\hline Non-Hispanic, Spanish or Latino & 179 & $(97.3 \%)$ & 86 & $(97.7 \%)$ & 53 & $(94.6 \%)$ & 40 & $(100 \%)$ \\
\hline Hispanic, Spanish or Latino & 5 & $(2.7 \%)$ & 2 & $(2.3 \%)$ & 3 & $(5.4 \%)$ & 0 & $(0 \%)$ \\
\hline \multicolumn{9}{|l|}{ Years providing healthcare services } \\
\hline Less than 5 years & 9 & $(4.8 \%)$ & 1 & $(1.1 \%)$ & 4 & $(7.0 \%)$ & 4 & $(10.0 \%)$ \\
\hline 6 - 9 years & 33 & $(17.5 \%)$ & 18 & $(19.8 \%)$ & 11 & $(19.3 \%)$ & 4 & $(10.0 \%)$ \\
\hline $10-14$ years & 47 & $(25.0 \%)$ & 23 & $(25.2 \%)$ & 16 & $(28.1 \%)$ & 8 & $(20.0 \%)$ \\
\hline 15 - 24 years & 52 & $(27.7 \%)$ & 27 & (29.7\%) & 13 & $(22.8 \%)$ & 12 & $(30.0 \%)$ \\
\hline $25+$ years & 47 & $(25.0 \%)$ & 22 & $(24.2 \%)$ & 13 & $(22.8 \%)$ & 12 & (30.0\%) \\
\hline \multicolumn{9}{|l|}{ Practice Setting } \\
\hline Urban & 103 & (54.8\%) & 38 & (41.8\%) & 34 & (59.6\%) & 31 & (77.5\%) \\
\hline Suburban & 86 & $(43.6 \%)$ & 50 & (54.9\%) & 23 & $(40.4 \%)$ & 9 & (22.5\%) \\
\hline Rural & 3 & $(1.6 \%)$ & 3 & (3.3\%) & 0 & $(0 \%)$ & 0 & $(0 \%)$ \\
\hline \multicolumn{9}{|l|}{ Years at present location } \\
\hline Less than 2 years & 16 & (8.5\%) & 5 & (5.5\%) & 7 & $(12.3 \%)$ & 4 & (10.0\%) \\
\hline 2 - 4 years & 52 & $(27.7 \%)$ & 23 & (25.3\%) & 15 & (26.3\%) & 14 & (35.0\%) \\
\hline 5 - 9 years & 52 & (27.7\%) & 26 & (28.5\%) & 12 & (21.0\%) & 14 & (35.0\%) \\
\hline 10 - 14 years & 32 & $(17.0 \%)$ & 14 & $(15.4 \%)$ & 14 & $(24.6 \%)$ & 4 & (10.0\%) \\
\hline $15+$ years & 36 & $(19.1 \%)$ & 23 & (25.3\%) & 9 & $(15.8 \%)$ & 4 & (10.0\%) \\
\hline
\end{tabular}

OB: obstetricians; FMP: family medicine physicians; CNM: certified nurse midwives. 
Steps were taken to ensure confidentiality of respondents who completed the survey on the internet. Study data were collected and managed using the Research Electronic Data Capture System (REDCap). REDCap is a secure, web-based application designed to support data capture for research studies [22]. Prior to completing the web-based survey, participants were presented with an information page which provided the informed consent form describing the purpose of the study, criteria for participation, confidentiality measures, incentive details, and contact information for the principal investigator and study coordinator. Participants were also reminded that their responses pertained to only pregnant women who were free of obstetric complications. Agreement to participate was confirmed by clicking on a "Continue" button which directed users to the survey. After completing the survey, participants were redirected to a separate, secure web page where they entered their name and e-mail address; this information was needed in order to receive the incentive (a $\$ 15$ gift card). Contact information was stored in a separate database such that it could not be linked to survey responses. Individuals who elected to complete the paper survey, provided a written informed consent and data were then entered using double data entry into the REDCap database.

\subsection{Data Management and Analyses}

Given the proposed comprehensive assessment, a survey was developed to assess HCPs' beliefs, attitudes, knowledge, and self-efficacy related to the counseling of antenatal physical activity. Attitudes toward antenatal physical activity focused on the physician's perceived level of responsibility toward providing counseling on antenatal physical activity (e.g. "It is an important responsibility of mine to discuss physical activity with my pregnant patients”). Physician beliefs related to benefits associated with antenatal physical activity (e.g. "Exercise during pregnancy improves the health for the mother"), their beliefs related to the safety of exercise during pregnancy (e.g. "Pregnant women should decrease their exercise as their pregnancy progresses"), and their beliefs related to who should exercise during pregnancy (e.g. "A sedentary woman, with an uncomplicated pregnancy, should not begin an exercise program during pregnancy”). The construct assessing the HCP's level of efficacy toward providing counseling on antenatal physical activity included items such as "I am confident in my knowledge regarding the benefits and risks of physical activity during pregnancy" and "I am confident in my ability to effectively talk with pregnant patients about antenatal physical activity”. HCP knowledge of antenatal physical activity pertained to their knowledge of the
2002 American Congress of Obstetricians and Gynecologists and the 2008 Department of Health and Human Services guidelines.

Perceived barriers toward providing antenatal physical activity counseling were also assessed (e.g. "To what extent does: 1) insufficient time; 2) inadequate knowledge; and 3) skills/uncertainty about available resources prevent you from administering physical activity counseling to your pregnant patients”). Current practices were captured by determining the percentage of patients who HCPs currently provide antenatal physical activity counseling to and in what form this is delivered: information (e.g. brochures, pamphlets, handouts, etc.), referrals, or in-office counseling by HCP or office staff. In addition, demographic and practice site information were collected such as age, gender, race, type of degree, type of healthcare provider, specialty area, years of practicing, and practice location (urban, suburban, rural). Last, we also assessed if and how healthcare providers would like future training related to antenatal physical activity counseling.

Recommended guidelines for developing survey questionnaires were utilized [23]. First, in order to create the survey an initial pool of approximately 75 items assessing the aforementioned constructs were developed. Some of the proposed items also were extracted from a preexisting survey "Exercise and Pregnancy Survey for Healthcare Providers” [18]. Constructs were conceptualized into two general classes which were treated differently throughout the survey development process. SingleItem traits were constructs that were presumed to be measured without error and which are only represented by a single item on the survey. Examples of single-item traits included sex, race, practice type, and other similar variables. Multi-item latent traits were measured with multiple survey items that were all presumed to measure an underlying trait with some measurement error. Examples of multi-item latent constructs include attitudes, beliefs, perceptions, knowledge, and general classes of behavior.

An expert panel of 11 HCPs (i.e. 3 OBs, 5 CNMs, 3 FMPs) were selected to perform the initial evaluation of the item pool. Panelists were asked to complete the online survey of 75 items and then asked to review, rate, and comment on all items in the initial pool. Participants were reminded before the onset of the survey that their responses pertained to only pregnant women who were free of obstetric complications. Panelists also provided qualitative feedback (e.g. wording changes, impressions, etc.) on individual items, survey sections, and the survey overall. Upon completion of this process, comments and feedback were transcribed and summarized for common themes. Single-item traits were revised based on commonalities across input from panelists. Any item that 
received a below-median average rating from panelists was rewritten or dropped from the item pool.

Multi-item traits were examined through standard item-scale diagnostics that included item distributional qualities, scale reliability, and item contributions to the scale (e.g., item-total correlations, reliability increases from each item). Items that were invariant, strongly skewed, showed prominent ceiling or floor effects, or which made negligible or negative contributions to scale reliability were dropped or rewritten. Panelist ratings of items were also considered in deciding item retention and drop decisions.

The final 39-item close-ended survey was then placed on a secured web-site designed for purposes of the proposed project. Consistent with current recommendations, the online survey was tested for user-friendliness [24]. To do so, a group of healthcare providers and researchers were asked to complete the web-based survey. The structure of the survey and whether it flows smoothly and in a logical manner, as well as survey completion time was assessed. During this pilot phase, written and verbal feedback was obtained and appropriate modifications were made resulting in a survey instrument that was self-administered in less than 10 minutes.

\subsection{Analyses}

Descriptive statistics for demographics and items for each construct were computed. One-way ANOVAs and chi-square tests of association were used, as appropriate, to explore potential differences on current healthcare provider characteristics (e.g. attitudes, beliefs, knowledge, current practices), and perceived barriers across the two specialties and healthcare provider types. All analyses were conducted using SAS Version 9.2.

\section{RESULTS}

Table 1 shows the descriptive characteristics of the study sample. More participants were trained as MDs specializing in obstetrics (48\%) while family medicine physicians and certified nurse midwives comprised smaller proportions of the sample, $30 \%$ and $21 \%$, respectively. The majority of the respondents were female $(71 \%)$, white (92\%), non-Hispanic (97\%), and practicing in an urban setting (55\%). Most participants had been providing healthcare for at least 10 years (78\%) and the majority (64\%) had been practicing in the DAMSA area for 5 years or more.

Table 2 displays the providers' beliefs regarding physical activity and their beliefs about their patients' responses to their advice. The majority of all providers agreed that physical activity during pregnancy will result in improved health for mother (i.e. 99\% agreed), baby (i.e. 89\% agreed), emotional wellbeing (i.e. 99\% agreed) and will reduce excessive weight gain (i.e. 96\% agreed). The majority of HCPs (i.e. 84\%) also believed that it is important to encourage their patients to meet ACOG's recommendation of at least 30 minutes of physical activity each day however there were significant differences across specialties with only $72 \%$ of FMPs agreeing. Unfortunately, only $43 \%$ of providers believed their patients followed the advice they are given about physical activity, despite a large portion (95\%) of providers reporting that it is important to discuss physical activity with their pregnant patients. Few providers reported that they felt that patients were uncomfortable talking about physical activity during pregnancy (7\%), but a greater portion responded that overweight patients are uncomfortable discussing physical activity with them (23\%). Over $40 \%$ of the sample was not confident that the information they distribute on physical activity was consistent with the current guidelines. Over $60 \%$ of the HCPs felt confident in their knowledge regarding the benefits and risks of physical activity, but there were significant differences across specialties with only $40 \%$ of FMPs being confident. Although the percentage of HCPs who believed sedentary, or sedentary and overweight patients should not begin exercise during pregnancy was very low (i.e., $3 \%$ and $1 \%$, respectively), HCPs reported lower confidence in effectively communicating with patients who are overweight and obese than patients of normal weight.

Data related to HCPs' current practices and training are provided in Table 3. HCPs as a group reported that they discuss antenatal physical activity with approximately two-thirds of their patients. However, these percentages differed across specialties with OBs and CNMs discussing antenatal physical activity with more patients than FMPs (i.e., 74\% OBs, 71\% CNMs, and 57\% FMPs; $\mathrm{p}=0.0028$ ). A similar pattern and difference by provider type was observed when examining the percent of providers that are likely to recommend a community physical activity program designed for pregnant women, with fewer FMPs being likely compared to OBs and CNMs ( $\mathrm{p}=0.039$ ). Approximately $40 \%$ of HCPs reported that they would be likely to advise sedentary pregnant patients to begin exercise. In their current practices, about a third of HCPs hand out information such as brochures and pamphlets. Few providers refer patients to an off-site physical activity resource and among specialties CNMs most frequently report referring their patients to an off-site community resource. Slightly over half of the providers reported that they provide in-office physical activity counseling, and FMs provide individualized counseling less often than OBs and CNMs (i.e. $33 \%, 60 \%$, and $65 \%$, respectively; $\mathrm{p}=0.0014$ ). The most commonly cited barrier to providing antenatal physical activity counseling was insufficient time. 
Table 2. Providers’ attitudes, beliefs, and efficacy: comparisons by specialty.

\begin{tabular}{|c|c|c|c|c|c|}
\hline & Full Sample & OB & FMP & CNM & $P$ value \\
\hline \multicolumn{6}{|l|}{ Attitudes ( $\%$ reporting that they $4=$ agree or $5=$ strongly agree) } \\
\hline Percentage that agree it is important to discuss PA with patients & $94.7 \%$ & $97.8 \%$ & $89.5 \%$ & $95.0 \%$ & 0.084 \\
\hline $\begin{array}{l}\text { Percentage that agree it is important to encourage patients to engage } \\
\text { in } 30 \text { minutes of PA each day }\end{array}$ & $84.6 \%$ & $89.0 \%$ & $71.9 \%$ & $92.5 \%$ & $0.006^{*}$ \\
\hline \multicolumn{6}{|l|}{ Beliefs (\% reporting that they $4=$ agree or 5 = strongly agree) } \\
\hline Sedentary, pregnant women should not begin an exercise program & $3.2 \%$ & $3.3 \%$ & $3.5 \%$ & $2.5 \%$ & 0.999 \\
\hline $\begin{array}{l}\text { Sedentary, overweight, pregnant women should not begin an exer- } \\
\text { cise program }\end{array}$ & $1.6 \%$ & $1.1 \%$ & $1.7 \%$ & $2.5 \%$ & 0.786 \\
\hline $\begin{array}{l}\text { Pregnant women should decrease their exercise as their pregnancy } \\
\text { progresses }\end{array}$ & $10.6 \%$ & $13.2 \%$ & $12.3 \%$ & $2.5 \%$ & 0.168 \\
\hline \multicolumn{6}{|c|}{ Percentage that agree that physical activity during pregnancy will result in } \\
\hline Improved health for mother & $99.5 \%$ & $98.9 \%$ & $100 \%$ & $100 \%$ & 0.484 \\
\hline Improved health for baby & $88.8 \%$ & $85.7 \%$ & $93.0 \%$ & $90.0 \%$ & 0.380 \\
\hline Improved emotional wellbeing & $99.5 \%$ & $100 \%$ & $100 \%$ & $97.5 \%$ & 0.213 \\
\hline Reduced excessive weight gain & $95.7 \%$ & $94.5 \%$ & $96.5 \%$ & $97.5 \%$ & 0.806 \\
\hline \multicolumn{6}{|l|}{ Patient Beliefs (\% reporting that they $4=$ agree or $5=$ strongly agree) } \\
\hline Patients follow the advice I give about PA & $43.1 \%$ & $41.8 \%$ & $43.9 \%$ & $45.0 \%$ & 0.933 \\
\hline Sedentary patients will not follow the advice to start engaging in PA & $22.3 \%$ & $24.2 \%$ & $21.0 \%$ & $20.0 \%$ & 0.836 \\
\hline Patients are uncomfortable talking about PA & $6.9 \%$ & $7.7 \%$ & $7.0 \%$ & $5.0 \%$ & 0.940 \\
\hline $\begin{array}{l}\text { Overweight patients are uncomfortable talking } \\
\text { about PA }\end{array}$ & $24.5 \%$ & $25.3 \%$ & $22.8 \%$ & $25.0 \%$ & 0.932 \\
\hline \multicolumn{6}{|l|}{ Self-Efficacy (\% reporting that they $4=$ agree or $5=$ strongly agree) } \\
\hline Confident in knowledge regarding the benefits and risks of PA & $63.8 \%$ & $75.8 \%$ & $40.4 \%$ & $70.0 \%$ & $<0.001^{*}$ \\
\hline $\begin{array}{l}\text { Confident that the information on PA that I distribute to my patients } \\
\text { is consistent with guidelines }\end{array}$ & $40.4 \%$ & $50.6 \%$ & $22.8 \%$ & $42.5 \%$ & $0.004^{*}$ \\
\hline $\begin{array}{l}\text { Confident in my ability to effectively talk with } \\
\text { patients about PA }\end{array}$ & $71.8 \%$ & $85.7 \%$ & $45.6 \%$ & $77.5 \%$ & $<0.001^{*}$ \\
\hline $\begin{array}{l}\text { Confident in my ability to effectively talk with } \\
\text { overweight and obese patients about PA }\end{array}$ & $61.2 \%$ & $70.3 \%$ & $30.3 \%$ & $21.3 \%$ & $0.011^{*}$ \\
\hline
\end{tabular}

OB: obstetricians; FMP: family medicine physicians; CNM: certified nurse midwives; ${ }^{*}$ significant, $P<0.05$.

Providers were tested on their knowledge of the current recommendations for physical activity for pregnant women as recommended by ACOG and DHHS. About 90\% ( $n=169)$ accurately described the types of exercises that are generally considered safe for pregnant women free of obstetric complications. Some providers ( $\mathrm{n}=16,9 \%$ ) incorrectly believed that running or weight bearing activities were not safe forms of physical activity. Nearly $30 \%(n=54)$ of respondents did not correctly identify the warning signs to terminate exercise while pregnant, yet $85 \%(n=159)$ were able to correctly identify ACOG's absolute contraindications to antenatal physical activity. Unfortunately, over half the sample $(55 \%, \mathrm{n}=102)$ incorrectly believed that a pregnant woman's heart rate should not exceed 140 beats/minute during exercise and just under half $(48 \%, \mathrm{n}=89)$ were not familiar with the current DHHS's recommendation that women free of obstetric complications should engage in at least 150 minutes of exercise per week. When stratified by provider type, there was a significant difference in the portion of providers that believed a pregnant woman's heart rate should not exceed 140 beats/min during exercise $(P=0.0175)$. The FMPs and CNMs more frequently agreed with this statement $(68 \%$ and $60 \%$, 
Table 3. Providers’ practices and barriers: comparisons by specialty.

\begin{tabular}{|c|c|c|c|c|c|}
\hline & Full Sample & OB & FMP & CNM & $P$ value \\
\hline Practices & Mean (SD) & Mean (SD) & Mean (SD) & Mean (SD) & \\
\hline $\begin{array}{l}\text { Percentage of pregnant patients that HCPs specifically discussed } \\
\text { exercise }\end{array}$ & $68.3(30.7)$ & $74.2(24.5)$ & $56.8(36.5)$ & $71.0(30.6)$ & $0.003^{*}$ \\
\hline $\begin{array}{l}\text { Percent likely to recommend a community physical activity } \\
\text { program designed for pregnant woman }\end{array}$ & $69.2 \%$ & $74.7 \%$ & $56.1 \%$ & $75.0 \%$ & $0.039^{*}$ \\
\hline Percent advising sedentary patients begin a moderate-intensity PA & $41.0 \%$ & $47.3 \%$ & $28.1 \%$ & $45.0 \%$ & 0.058 \\
\hline \multicolumn{6}{|l|}{ Indicated utilizing method of providing information on PA } \\
\hline Information (i.e. brochures, pamphlets, handouts) & $28.7 \%$ & $27.5 \%$ & $26.3 \%$ & $35.0 \%$ & 0.606 \\
\hline Refer patient to an off-site physical activity community resource & $11.2 \%$ & $9.9 \%$ & $5.3 \%$ & $20.5 \%$ & $0.026^{*}$ \\
\hline $\begin{array}{l}\text { Counseling (i.e. provide individualized attention to a patient } \\
\text { regarding their physical activity) in your office by you }\end{array}$ & $53.2 \%$ & $60.4 \%$ & $33.3 \%$ & $65.0 \%$ & $0.001^{*}$ \\
\hline Counseling in your office by another health care provider & $74.6 \%$ & $76.9 \%$ & $69.6 \%$ & $74.4 \%$ & 0.619 \\
\hline \multicolumn{6}{|l|}{ Indicated item as a barrier } \\
\hline Insufficient time & $22.5 \%$ & $17.8 \%$ & $29.8 \%$ & $22.7 \%$ & 0.234 \\
\hline Inadequate knowledge/skills & $3.7 \%$ & $2.2 \%$ & $7.0 \%$ & $2.3 \%$ & 0.280 \\
\hline Uncertainty about available resources & $10.5 \%$ & $5.6 \%$ & $12.3 \%$ & $18.2 \%$ & 0.070 \\
\hline
\end{tabular}

OB: obstetricians; FMP: family medicine physicians; CNM: certified nurse midwives; ${ }^{*}$ significant, $P<0.05$.

respectively) while less than half $(44 \%)$ of the OBs agreed $(P=0.0167)$. Importantly, $17 \%(\mathrm{n}=31)$ of providers reported that they never received professional training in antenatal physical activity counseling. Of those that reported receiving training, only $31 \%(n=48)$ reported that the training was "good" or "excellent", while the majority, $69 \%(\mathrm{n}=107)$ claimed their training was "fair" or "poor".

\section{DISCUSSION}

The Department of Health and Human Services (DHHS) recommends that adults engage in a total of 150 minutes of moderate-intensity exercise each week. DHHS expanded the national guidelines on physical activity to include pregnant women and recommended that pregnant women free of obstetric complications engage in moderate-intensity physical activity on all or most days of the week [1]. The American Congress of Obstetricians and Gynecologists (ACOG) concurs that pregnant women free of obstetric complications engage in regular physical activity throughout their pregnancy and that moderateintensity physical activity should be performed on most or all days of the week [25]. Unfortunately, many women fail to engage in regular physical activity during pregnancy and often times it is due to uncertainty about the safety of exercise during pregnancy and the knowledge needed to make informed decisions about exercise. Healthcare providers (HCPs) play an integral role in pro- moting healthy lifestyle behaviors, such as physical activity, during pregnancy. Especially given that research suggests that the majority of pregnant women indicate that their HCP has great influence on their beliefs regarding behavioral change and that brief counseling on behavioral change by HCPs increases the likelihood that patients will adopt behavior $[15,16]$. Thus, HCPs are a knowledgeable source of information for women, particularly during a major life transition such as pregnancy. To date there is a dearth of literature examining HCPs' attitudes, beliefs, and current practices pertaining to antenatal physical activity. The present cross-sectional study addressed this gap by providing a comprehensive assessment of three types of HCPs' (i.e. obstetricians, family medicine practitioners, and certified nurse midwives) attitudes, beliefs, perceived barriers and current practices related to antenatal physical activity in order to help inform the development of future intervention studies.

Consistent with previous research, findings from the present study suggest that the majority of HCPs across all three specialties believe engagement in antenatal physical activity will result in improved health for mother and baby [18,20,21]. Moreover, most of the HCPs agree that it is important that pregnant women free of obstetric complications should meet the DHHS recommendations on antenatal physical activity. However, as a group, HCPs reported not discussing antenatal physical 
activity with approximately one third of their pregnant patients which is consistent with previous studies [17, $19,21]$. Moreover, only $41 \%$ of the HCPs stated they advise sedentary pregnant patients to begin engaging in regular physical activity. Surprisingly FMPs were less likely than obstetricians or nurse midwives to discuss antenatal physical activity with their patients. This may be due in part to previous training on antenatal physical activity as approximately $30 \%$ of the FMPs reported their training as "poor" compared to $13 \%$ for obstetricians and nurse midwives.

Clearly, there is a disconnect between translating the knowledge of the importance of antenatal physical activity and current guidelines into practice. There are a few potential reasons for this disconnect. First, it may be due to perceived patient barriers. For example, our findings suggest that over half of HCPs believe that the patient will not follow the physical activity advice given and a quarter of the HCPs believe overweight patients are uncomfortable talking about antenatal physical activity. Future research is warranted to better understand HCPs' beliefs about patient adherence to recommended behavioral change.

The low levels of antenatal physical activity counseling may also be due to the lack of knowledge pertaining to antenatal physical activity among HCPs. Although most providers correctly identified safe exercises and recognized the absolute contraindications to exercise, they lacked the knowledge about current guidelines specific to recommended exercise prescription (e.g. mode, frequency, intensity and duration of antenatal physical activity). For example, our findings suggest that just under half of the HCPs agreed with the outdated 1985 ACOG recommendations that a pregnant woman's heart rate should not exceed 140 beats/minute [26]. These findings as well as others $[17,18]$ imply that there is a need to increase awareness of the current ACOG recommendations more comprehensively. Moreover, almost half of the sample was not familiar with DHHS's recommendation to engage in at least 150 minutes of moderate-intensity exercise per week. A similar lack of knowledge regarding the current recommendations for physical activity has been found in primary care settings [27]. A large portion of the respondents in the present survey (43\%) had been practicing health care for greater than 18 years and may not have received training regarding the most recent ACOG recommendations, underscoring the need for continuing education opportunities in this area.

Our study results showed over a third of the HCPs were not confident in their knowledge regarding the benefits and risks of antenatal physical activity and over a quarter reported that they were not confident in their ability to effectively talk with patients about antenatal physical activity. Moreover, this confidence was lower talking with overweight or obese patients which may be due to their perceptions that overweight and obese pregnant patients are uncomfortable talking about pregnant patients. Given that knowledge and skill-building are often associated with increased confidence to change a given health behavior [28], in this case providing antenatal physical activity counseling, these areas should be targeted in future educational efforts.

Last, it may also be due to perceived practice-level barriers such as limited time and uncertainty about available resources. Similar to previous studies, insufficient time was not surprisingly reported as one of the main barriers to providing physical activity counseling [27]. Although, many providers stated that antenatal physical activity counseling is provided in their office, but by another provider. While insufficient time could prevent HCPs from providing individual in-person counseling, it seems reasonable that distributing information or referring patients to community resources would be viable alternative options. However, our findings suggest that few HCPs provide handouts or outsource to community outlets. Moreover, if HCPs do distribute handouts, the information may be outdated as findings from the present study suggest that many HCPs (approximately 60\%) are not confident that the material they currently distribute to patients appropriately reflects current guidelines on antenatal physical activity. These findings highlight the importance of increasing HCPs awareness of community resources designed to support exercise strategies for pregnant women as well as access to updated and relevant handouts.

\subsection{Limitations}

The present study employed the recommended approaches from previous research involving HCPS surveillance studies [29], such as using monetary incentives, brief questionnaires and multiple follow-up attempts. Specifically the study utilized the directors of local chapters/ organizations, who have primary contact for numerous healthcare providers throughout the DAMSA, by having them distribute the pre-notification and subsequent invitations to participate in the survey to all eligible healthcare providers. Despite these efforts our response rate was lower than expected. This may have been due in part to budget limitations and the inability to provide adequate compensation or the mere lack of HCP awareness of antenatal physical activity current guidelines and associated benefits in general. Findings from this study are also based on a small, geographical sample and thus, may not represent other populations. Last, due to the nonrandomization of the sample, response bias may also be a limitation. Moreover, there may be selectivity bias such that only those HCPs who were interested in the 
topic of antenatal physical activity agreed to participate in the study.

\subsection{Practical Implications}

These data provide insight to tailor future approaches aimed at enhancing antenatal physical activity counseling in primary care settings. In order to provide accurate and effective counseling, HCPs must be aware of the most current recommendations, the benefits of, and the risks to engaging in physical activity during pregnancy. Findings from the present study demonstrate a clear need for further continuing education opportunities on the current guidelines on antenatal physical activity; which in turn, may positively impact the likelihood and overall quality of antenatal physical activity counseling among HCPs.

\section{REFERENCES}

[1] Zhang, J. and Savitz, D.A. (1996) Exercise during pregnancy among US women. Annals of Epidemiology, 6, 53-59. doi:10.1016/1047-2797(95)00093-3

[2] Evenson, K.R., Savitz, D.A. and Huston, S.L. (2004) Leisure-time physical activity among pregnant women in the US. Paediatric and Perinatal Epidemiology, 18, 141149. doi:10.1111/j.1365-3016.2004.00595.x

[3] Poudevigne, M.S. and O’Connor, P.J. (2006) A review of physical activity patterns in pregnancy women and their relationship to psychological health. Sports Medicine, 36, 19-38. doi:10.2165/00007256-200636010-00003

[4] Collings, C.A., Curet, L.B. and Mullin, J.P. (1983) Maternal and fetal responses to a maternal aerobic exercise program. American Journal of Obstetrics \& Gynecology, 145, 702-707.

[5] Clapp III, J.F. (1990) The course of labor after endurance exercise during pregnancy. American Journal of Obstetrics \& Gynecology, 163, 1799-1805.

[6] Sternfeld, B., Quesenberry, C.P. and Eskenazi, B. (1995) Exercise during pregnancy and pregnancy outcome. MSSE, 27, 634-640.

[7] Da Costa, D., Rippen, N., Dritsa, M. and Ring, A. (2003) Self-reported leisure-time physical activity during pregnancy an relationship to psychological well-being. Journal of Psychosomatic Obstetrics \& Gynecology, 24, 111119.

[8] US Department of Health and Human Services (2008) Physical Activity Guidelines for Americans.

[9] Galper, D.I., Trivedi, M.H., Barlow, C.E., Dunn, A.L. and Kampert, J.B. (2006) Inverse association between physiccal inactivity and mental health in men and women. MSSE, 38, 173-178.

[10] Marcus, B.H., Williams, D.M., Dubbert, P.M., Sallis, J.F., King, A.C., Yancey, A.K., Franklin, B.A., Buchner, D., Daniels, S.R. and Claytor, R.P. (2006) Physical activity intervention studies: What we know and what we need to know: A scientific statement from the American Heart
Association Council on Nutrition, Physical Activity, and Metabolism (Subcommittee on Physical Activity); Council on Cardiovascular Disease in the Young; and the Interdisciplinary Working Group on Quality of Care and Outcomes Research. Circulation, 114, 2739-2752. doi:10.1161/CIRCULATIONAHA.106.179683

[11] Manson, J.E., Hu, F.B. and Rich-Edwards, J.W. (1999) A prospective study of walking as compared with vigorous exercise in the prevention of coronary heart disease. New England Journal of Medicine, 341, 650-658. doi:10.1056/NEJM199908263410904

[12] Clarke, P.E. and Gross, H. (2004) Women's behaviour, beliefs and information sources about physical exercise in pregnancy. American Journal of Obstetrics \& Gynecology, 20, 133-141.

[13] Kieffer, E.C., Willis, S.K., Arellano, N. and Guzman, R. (2002) Perspectives of pregnant and postpartum latino women on diabetes, physical activity and health. Health Education \& Behavior, 29, 542-556. doi:10.1177/109019802237023

[14] Doran, F. and O’Brien, A.P. (2007) A brief report of attitudes towards physical activity during pregnancy. Australian Health Promotion Association, 18, 155-158.

[15] Krans, E.E. and Gearhart, J.G. (2005) Pregnant women's beliefs and influences regarding exercise during pregnancy. Journal of the Mississippi State Medical Association, 46, 67-73.

[16] Aittasalo, M., Pasanen, M., Fogelholm, M., Kinnunen, T.I., Ojala, K. and Luoto, R. (2008) Physical activity counseling in maternity and child health care-A controlled trial. BMC Women's Health, 8, 14. doi:10.1186/1472-6874-8-14

[17] Entin, P.L. and Munhall, K.M. (2006) Recommendations regarding exercise during pregnancy made by private/ small group practice obstetricians in the USA. Journal of Sports Science and Medicine, 5, 449-458.

[18] Bauer, P.W., Broman, C.L. and Pivarnik, J.M. (2004) Exercise and pregnancy survey for health care providers. MSSE, 36, S113.

[19] Herring, S.J., Platek, D.N., Elliot, P., Riley, L.E., Stuebe, A.M. and Oken, E. (2010) Addressing obesity in pregnancy: What do obstetric providers recommend? Journal of Women's Health, 19, 65-70. doi:10.1089/jwh.2008.1343

[20] Hughes, R., Maher, J., Baillie, E. and Shelton, D. (2011) Nutrition and physical activity guidance for women in the pre- and post-natal period: a continuing education needs assessment in primary health care. Australian Journal of Primary Health, 17, 135-141. doi:10.1071/PY10012

[21] Power, M.L., Cogswell, M.E. and Schulkin, J. (2006) Obesity prevention and treatment practices of US obstetrician-gynecologists. Obstetrics \& Gynecology, 108, 961968. doi:10.1097/01.AOG.0000233171.20484.db

[22] Harris, P.A., Taylor, R., Thielke, R., Payne, J., Gonzales, N. and Conde, J.G. (2009) A metadata-driven methodology and work flow process for providing translational research informatics support. Journal of Biomedical Informatics, 42, 377-381. doi:10.1016/j.jbi.2008.08.010

[23] Presser, S., Rothgeb, J., Couper, M.P., et al. (2004) 
Methods for testing and evaluating survey questionnaires. John Wiley \& Sons, Hoboken. doi:10.1002/0471654728

[24] Daley, E.M., McDermott, R.J., McCormack-Brown, K.R. and Kittleson, M.J. (2003) Conducting web-based survey research: A lesson in internet designs. American Journal of Health Behavior, 27, 116-124. doi:10.5993/AJHB.27.2.3

[25] ACOG (2002) ACOG committee opinion: Exercise during pregnancy and postpartum period. Obstetrics \& Gynecology, 99, 171-173. doi:10.1016/S0029-7844(01)01749-5

[26] American Congress of Obstetricians and Gynecologists (1985) Women and ExerciseExercise during pregnancy and the postnatal period. ACOG, Washington.

[27] Douglas, F., Torrance, N., van Teijlingen, E., Meloni, S. and Kerr, A. (2006) Primary care staff's views and experiences related to routinely advising patients about physical activity. A questionnaire survey. BMC Public Health, 6, 138-148. doi:10.1186/1471-2458-6-138

[28] Glanz, K., Rimer, B.K. and Viswanath, K., Eds. (2008) Health Behavior and Health Education. 4th Edition, Jossey-Bass Publishers, San Franciso.

[29] Easton, A.N., Price, J.H. and Telljohann, S.K. (1997) An informational versus monetary incentive in increasing physicians' response rates. Psychological Reports, 81, 968-970. doi:10.2466/pr0.1997.81.3.968 